\title{
An orlistat "overdose" in a child
}

\author{
M. B. O'Connor
}

Received: 16 July 2009/Accepted: 20 August 2009/Published online: 11 September 2009

(C) Royal Academy of Medicine in Ireland 2009

Dear Editor,

I would like to present the case of a 28-month-old girl who recently presented to the Accident and Emergency Department of our General Hospital following the accidental ingestion of $5,160 \mathrm{mg}$ of orilstat $\left(\mathrm{Alli}^{\circledR}\right)$. Her mother, who was taking this newly available over-the-counter medication for weight loss, found the child on the living room floor with the open medication bottle and a number of capsules open on the floor. In total 43 tablets were unaccounted for, at a dose of $120 \mathrm{mg}$ per tablet. On presentation to the accident and Emergency Department, 20 min following the incident, the young girl was alert, in no physical distress and completely asymptomatic.

We contacted our national toxicology centre for advice. Unfortunately reported cases of orilstat exposure in children are limited [1]. However, based on product information we were advised to monitor the girl for $6 \mathrm{~h}$ with particular focus on GI symptoms. Fatty foods were advised against for $24 \mathrm{~h}$. If symptoms developed supportive measures were recommended. Thankfully this young girl remained asymptomatic for that period. She was subsequently reviewed by a nearby Accident and Emergency Pediatrics service, following discharge from our hospital, and cleared by them with no resultant consequences from her overdose.

Orlistat (marketed as a prescription only medicine as Xenical ${ }^{\circledR}$ by Roche; or over-the-counter as Alli ${ }^{\circledR}$ by GlaxoSmithKline), also known as tetrahydrolipstatin, is a drug designed to treat obesity. Its primary function is preventing the absorption of fats from the human diet, thereby reducing caloric intake. It is intended for use in conjunction with a physiciansupervised reduced-calorie diet. Orlistat is the saturated

M. B. O'Connor ( $\square)$

Department of Medicine, Mallow General Hospital,

Mallow, Ireland

e-mail: mortimeroconnor@gmail.com derivative of lipstatin, a potent natural inhibitor of pancreatic lipases isolated from the bacterium Streptomyces toxytricini [2]. However, due to simplicity and stability, orlistat rather than lipstatin was developed into an anti-obesity medication.

Orlistat works by inhibiting gastrointestinal lipase, an enzyme that breaks down triglycerides in the intestine. Without this enzyme, triglycerides from the diet are prevented from being hydrolyzed into absorbable free fatty acids and are excreted undigested. Only trace amounts of orlistat are absorbed systemically; the primary effect is local lipase inhibition within the GI tract after an oral dose. The primary route of elimination is through the feces. At the standard prescription dose of $120 \mathrm{mg}$ three times daily before meals, orlistat prevents approximately $30 \%$ of dietary fat from being absorbed. Higher doses do not produce more potent effects.

The primary side effects of the drug are gastrointestinalrelated, and include steatorrhea, fecal incontinence, frequent or urgent bowel movements, and flatulence. To minimize these effects, foods with high fat content should be avoided; the manufacturer advises consumers to follow a low-fat, reducedcalorie diet. Oily stools and flatulence can be controlled by reducing the dietary fat content to somewhere in the region of $15 \mathrm{~g}$ per meal.

With Alli ${ }^{\circledR}$ now being available over the counter, fears regarding overdose can be somewhat reassured by this case where a 28-month-old had a large ingestion of the medication without adverse effects.

\section{References}

1. Forrester MB (2008) Pattern of orlistat exposures in children aged 5 years or less. J Emerg Med Apr 8. [Epub ahead of print] PMID: 18403165

2. Barbier P, Schneider F (1987) Syntheses of tetrahydrolipstatin and absolute configuration of tetrahydrolipstatin and lipstatin. Helv Chim Acta 70(1):196-202 